
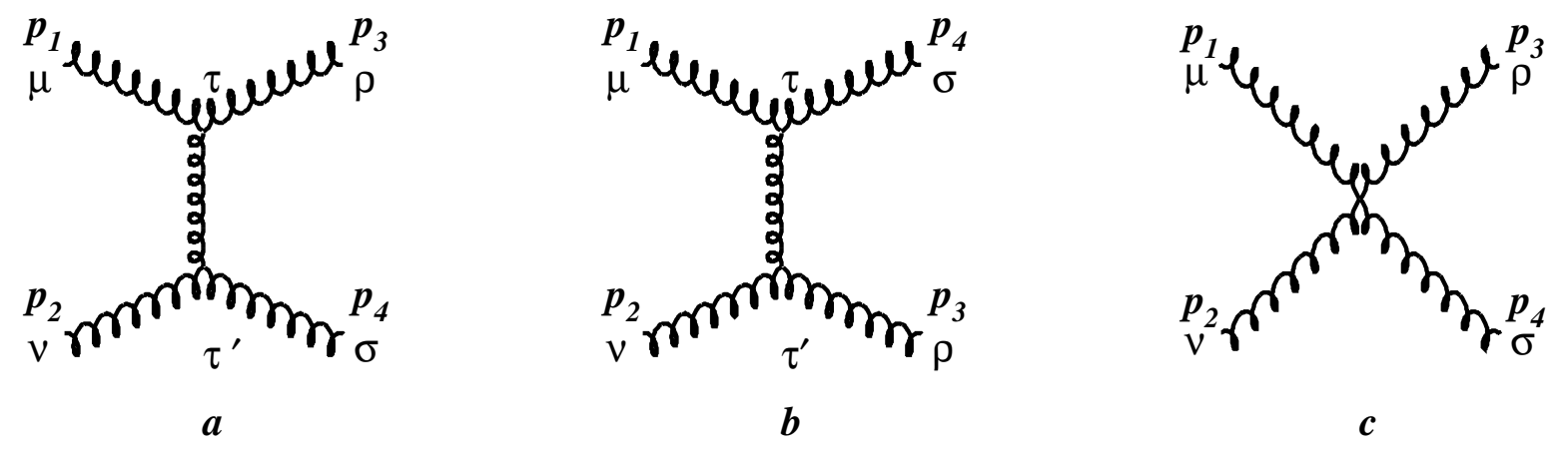

Fig. 1 
BIHEP-TH-97-011

\title{
Glueball Spectrum from the B.S. Equation
}

\author{
J.Y. Cui ${ }^{a}{ }^{b}$ J.M. Wu ${ }^{a}$ and H.Y. Jin ${ }^{a}$ \\ ${ }^{a}$ Institute of High Energy Physics \\ Beijing, 100039, P.R. China \\ ${ }^{b}$ Department of Physics, Henan Normal University \\ Xinxiang, 453002, P.R. China
}

\begin{abstract}
The mass of the glueballs is calculated in the B.S. equation framework. Under instantaneous approximation, the wave function of B.S. equations are obtained. The kernel is chosen as the sum of an one-gluon exchange potential, a contact interaction and a linear confining potential. The numerical results are in agreement with that of recent lattice calculation.
\end{abstract}

PACS: 14.40.C, 11.10.St.

Keywords: glueball, B.S. equation. 
1.Introduction The existence of glueball states is a very important prediction of QCD, whose discovery would be a direct confirmation of the non-abelian (self-coupling) character of the gluonic degree of freedom in strong interactions. Therefore, these states are in focus of the interest of both theorists and experimentalists. Glueballs were suggested by Gell-Mann and Fritzsch [1], Fritzsch and Minkowski[2]. Since then a lot of investigation has been made. There are various theoretical approaches applied, which

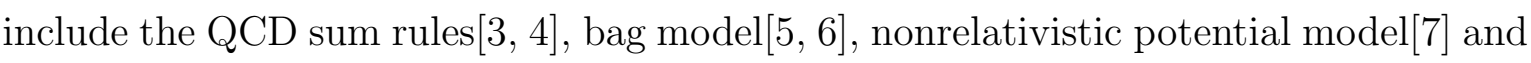
lattice calculation [8, 9]. However, these approaches differ from each other markedly in their mass prediction, sometimes as large as $1 \mathrm{GeV}$. Therefore, it's necessary to make more investigations.

On the other hand, in relativistic quantum field theory, the Bethe-Salpeter(B.S.) equation is an exact bound state equation. With some appropriate approximation it has been used successfully in the study of $q \bar{q}$ bound states [10]. Therefore, it would be reasonable for us to try to study the bound states of the gluons in the framework of B.S. equation. In our investigation, the B.S kernel is chosen as the sum of an onegluon exchange potential, a contact interaction and a linear confining potential. We calculate the mass of $0^{++}, 2^{++}, 0^{-+}$and $2^{-+}$glueballs. The numerical results are in good agreement with that of recent lattice calculation.

The paper is arranged as follows: in the next section, we construct the B.S. equation for the bound states of two constituent gluons, and then we discuss the integral kernel and the structure of the B.S. wave functions of glueballs investigated. In section three the numerical results and a discussion are presented.

2.The B.S. Equation for Glueballs Let $A_{\mu}^{a}\left(x_{1}\right)$ and $A_{\nu}^{b}\left(x_{2}\right)$ be the gluon fields at points $x_{1}$ and $x_{2},|G\rangle$ the bound state of two gluons with mass $M$ and momentum $P_{\mu}$. Then the B.S. wave function for a bound state is defined as

$$
\chi_{\mu \nu}^{a b}\left(P, x_{1}, x_{2}\right)=\left\langle 0\left|T\left(A_{\mu}^{a}\left(x_{1}\right) A_{\nu}^{b}\left(x_{2}\right)\right)\right| G\right\rangle,
$$

where $\mu, \nu$ are Lorentz indices, and $a, b$ color indices. The glueballs are color singlet 
states, so we have

$$
\chi_{\mu \nu}^{a b}\left(P, x_{1}, x_{2}\right)=\delta^{a b} \chi_{\mu \nu}\left(P, x_{1}, x_{2}\right)
$$

The translation invariance of system implies

$$
\chi_{\mu \nu}\left(P, x_{1}, x_{2}\right)=e^{i P \cdot X} \chi_{\mu \nu}(P, x),
$$

where $X=\frac{x_{1}+x_{2}}{2}$ and $x=x_{1}-x_{2}$. We further define the B.S. wave function in momentum space as

$$
\chi_{\mu \nu}(P, q)=\int \frac{d^{4} x}{(2 \pi)^{4}} e^{-i q x} \chi_{\mu \nu}(P, x),
$$

where $q$ is the relative momentum of the two constituent gluons.

With a standard method we obtain the B.S. equation for a color singlet glueball state,

$$
\chi_{\mu \nu}(P, q)=\Delta_{\mu \alpha}\left(p_{1}\right) \Delta_{\nu \beta}\left(p_{2}\right) \int \frac{d^{4} k}{(2 \pi)^{4}} G^{\alpha \beta \rho \sigma}(P, q, k) \chi_{\rho \sigma}(P, k),
$$

where the color indices have been suppressed. The tensor kernel $G^{\alpha \beta \rho \sigma}(P, q, k)$ in the above equation is defined as the sum of all two-particle irreducible graphs, and $\Delta_{\mu \alpha}\left(p_{i}\right)$ is the full propagator of the constituent gluons with momentum $p_{i}$, and

$$
\begin{gathered}
P=p_{1}+p_{2}, \\
2 q=p_{1}-p_{2},
\end{gathered}
$$

where $P^{2}=M^{2}$.

Although this equation is formally exact, it is difficult to use in concrete problems. The reason is twofold: first we don't know how to calculate the kernel of B.S. equation, and second, even if we know the kernel we would be unable to solve the equation exactly. Therefore, to make the approximation is inevitable.

In solving the B.S. equation of glueballs, we make "static" approximation as usually do in the case of $q \bar{q}$ mesons. In this approximation one neglects the dependence of the kernel $G(P, q, k)$ on $k_{0}$ and $q_{0}$. Then the $d k_{0}$ integration on the right-hand side of equation (5) yields the equal time wave function

$$
\varphi_{\mu \nu}(P, \mathbf{k}) \equiv \int d k_{0} \chi_{\mu \nu}(P, k)
$$


Next we perform $d q_{0}$ integration on both sides of equation (5), and at the left-hand side this again yields the three dimensional wave function $\varphi_{\mu \nu}(P, \mathbf{q})$. On the right-hand side, the $q_{0}$ dependence is only contained in the variables $p_{10}$ and $p_{20}$. That is

$$
\varphi_{\mu \nu}(P, \mathbf{q})=\int d q_{0} \Delta_{\mu \alpha}\left(p_{1}\right) \Delta_{\nu \beta}\left(p_{2}\right) \int \frac{d^{3} k}{(2 \pi)^{4}} G^{\alpha \beta \rho \sigma}(P, \mathbf{q}, \mathbf{k}) \varphi_{\rho \sigma}(P, \mathbf{k}) .
$$

To go on, an appropriate gauge has to be fixed in equation (9). It's convenient for us to choose Coulomb gauge. In Coulomb gauge the propagator is

$$
\Delta_{\mu \nu}(k)=-\frac{i}{k^{2}+i \epsilon}\left[g_{\mu \nu}-\frac{1}{\mathbf{k}^{2}}\left(k_{\mu} k_{\nu}-k_{\mu} s_{\nu}-k_{\nu} s_{\mu}\right)\right]
$$

where

$$
s_{\mu} \equiv(0, \mathbf{k})
$$

We can write the propagator more clearly as

$$
\begin{gathered}
\Delta_{i j}(k)=\frac{i}{k^{2}+i \epsilon}\left(\delta_{i j}-\frac{k_{i} k_{j}}{\mathbf{k}^{2}}\right), \\
\Delta_{00}(k)=\frac{i}{\mathbf{k}^{2}} \\
\Delta_{0 i}(k)=\Delta_{i 0}(k)=0 .
\end{gathered}
$$

From (9) and (11), one finds that( for $\epsilon_{\mu}$, the polarization vector of the constituent gluons, we set $\epsilon_{0}=0$ )

$$
\varphi_{0 i}=\varphi_{i 0}=\varphi_{00}=0
$$

Therefore, in Coulomb gauge one need only deal with the three dimensional wave functions $\varphi_{i j}(P, \mathbf{q})(i=1,2,3)$. It's appropriate to give an effective mass $m$ to the constituent gluons and rewrite the propagator as

$$
\Delta_{i j}(k)=\frac{i}{k^{2}-m^{2}+i \epsilon}\left(\delta_{i j}-\frac{k_{i} k_{j}}{\mathbf{k}^{2}}\right) .
$$

For convenience, we adopt the center-of-mass system in the present paper. In this frame we have

$$
\int \frac{d q_{0}}{\left(p_{1}^{2}-m^{2}+i \epsilon\right)\left(p_{2}^{2}-m^{2}+i \epsilon\right)}=\frac{2 \pi i}{E\left(4 E^{2}-M^{2}\right)}
$$


where $E=\sqrt{m^{2}+\mathbf{q}^{2}}$. Substituting (16) and (17) into equation (9), we obtain

$$
\begin{gathered}
E\left(M^{2}-4 E^{2}\right) \varphi_{i j}(P, \mathbf{q})= \\
i\left(\delta_{i i^{\prime}}-\frac{q_{i} q_{i^{\prime}}}{\mathbf{q}^{2}}\right)\left(\delta_{j j^{\prime}}-\frac{q_{j} q_{j^{\prime}}}{\mathbf{q}^{2}}\right) \int \frac{d^{3} k}{(2 \pi)^{3}} G_{i^{\prime} j^{\prime} k l}(P, \mathbf{q}, \mathbf{k}) \varphi_{k l}(P, \mathbf{k}) .
\end{gathered}
$$

This equation is the starting point of our numerical investigation.

The next question is how to construct the integral kernel. We assume that the kernel $G_{i j k l}(P, \mathbf{q}, \mathbf{k})$ is the sum of two parts: the short distance part $G_{i j k l}^{(s)}(P, \mathbf{q}, \mathbf{k})$, and long distance part $G_{i j k l}^{(l)}(P, \mathbf{q}, \mathbf{k})$. As usual,we assume that the short distance part, $G_{i j k l}^{(s)}(P, \mathbf{q}, \mathbf{k})$, is contributed by the three lowest order diagrams shown in figure 1 . Besides equations (6) and (7), we also have

$$
\begin{gathered}
P=p_{3}+p_{4}, \\
2 k=p_{3}-p_{4} .
\end{gathered}
$$

Calculating diagram $a, b$ and $c, G_{\mu \nu \rho \sigma}^{(s)}(P, q, k)$ can be expressed explicitly as

$$
\begin{aligned}
G_{\mu \nu \rho \sigma}^{(s)}(P, q, k) & =3 i\left(4 \pi \alpha_{s}\right)\left\{2 C_{\mu \rho \tau}\left(p_{1}, p_{3}\right) C_{\nu \sigma \tau^{\prime}}\left(p_{2}, p_{4}\right)\left[\frac{g^{\tau 0} g^{\tau^{\prime} 0}}{\mathbf{l}^{2}}+\frac{g^{\tau i} g^{\tau^{\prime} j}}{l^{2}}\left(\delta_{i j}-\frac{l_{i} l_{j}}{\mathbf{l}^{2}}\right)\right]\right. \\
& \left.-\left(2 g_{\mu \nu} g_{\rho \sigma}-g_{\mu \rho} g_{\nu \sigma}-g_{\mu \sigma} g_{\nu \rho}\right)\right\}
\end{aligned}
$$

where $l(=q-k)$ is the momentum exchanged between the two constituent gluons, and

$$
\begin{gathered}
C_{\mu \rho \tau}\left(p_{1}, p_{3}\right)=\left(p_{1}-2 p_{3}\right)_{\mu} g_{\rho \tau}+\left(p_{1}+p_{3}\right)_{\tau} g_{\mu \rho}+\left(p_{3}-2 p_{1}\right)_{\rho} g_{\mu \tau}, \\
C_{\nu \sigma \tau^{\prime}}\left(p_{2}, p_{4}\right)=\left(p_{2}-2 p_{4}\right)_{\nu} g_{\sigma \tau^{\prime}}+\left(p_{2}+p_{4}\right)_{\tau^{\prime}} g_{\nu \sigma}+\left(p_{4}-2 p_{2}\right)_{\sigma} g_{\nu \tau^{\prime}} .
\end{gathered}
$$

The factor 3 on the right-hand side of equation (21) is the color factor which is $\frac{4}{3}$ in the case of $q \bar{q}$ bound state. Diagram $a$ and diagram $b$ make the same contribution to physical states, so there is a factor 2 in equation (21). The strong coupling constant $\alpha_{s}$ is chosen as a running one,

$$
\alpha_{s}=\frac{12 \pi}{27} \frac{1}{\ln \left(a+\frac{l^{2}}{\Lambda_{Q C D}^{2}}\right)},
$$


where $a$ is a parameter introduced to avoid the infrared divergence. As for the long distance part $G_{i j k l}^{(l)}$, we have no reliable knowledge about it, so we have to constructed it phenomenally. The experience with $q \bar{q}$ bound state shows that the long distance part and short distance part of kernel have different spin dependence. Generally speaking, the long distance part makes no contribution to spin-spin interaction. With the guidance of this experience, we only chose in equation (22-23) the terms containing tensor $g_{\mu \rho} g_{\nu \sigma}$ as the spin dependence of the confining part because such terms have nothing to do with spin effect. Therefore we assume

$$
G_{\mu \nu \rho \sigma}^{(l)}=2 i\left(p_{1}+p_{3}\right) \cdot\left(p_{2}+p_{4}\right) g_{\mu \rho} g_{\nu \sigma} G(l),
$$

where $G(l)$ is spatial dependence of confining part of the kernel. We choose

$$
G(l)=\frac{8 \pi \lambda}{l^{4}}
$$

which corresponds to a linearly growing potential. The expression $\frac{1}{l^{4}}$ is very singular at the zero point of $l$, and regularization is necessary. The method is to subtract a $\delta$ function from the confining part of the kernel, that is to make the following replacement,

$$
\begin{aligned}
G_{\mu \nu \rho \sigma}^{(l)} & =2 i\left(p_{1}+p_{3}\right) \cdot\left(p_{2}+p_{4}\right) g_{\mu \rho} g_{\nu \sigma} \frac{8 \pi \lambda}{\left(l^{2}+u^{2}\right)^{2}} \\
& -\delta^{3}(\mathbf{l}) \int d^{3} k\left\{2 i\left(p_{1}+p_{3}\right) \cdot\left(p_{2}+p_{4}\right) g_{\mu \rho} g_{\nu \sigma} \frac{8 \pi \lambda}{\left(l^{2}+u^{2}\right)^{2}}\right\}
\end{aligned}
$$

where $u$ is a small quantity. In actualcalculation we let $u \rightarrow 0$. In this way the infrared divergence is subtracted out.

The next important question is to construct B.S. wave function for a given gluon bound state. For $0^{-+}$glueball the most general decomposition of the four dimensional wave function is given by the following expression

$$
\chi_{\mu \nu}(P, q)=f \epsilon_{\mu \nu \alpha \beta} P^{\alpha} q^{\beta} .
$$

For $0^{++}$glueball,

$$
\chi_{\mu \nu}(P, q)=f_{0} g_{\mu \nu}+f_{1} P_{\mu} P_{\nu}+f_{2} P_{\mu} q_{\nu}+f_{3} P_{\nu} q_{\mu}+f_{4} q_{\mu} q_{\nu},
$$


where $f$ and $f_{i}$ are scalar functions of $P^{2}, q^{2}$ and $P \cdot q$. For other states the B.S. wave functions are more complicated and are not given here. Except for pseudoscalar state, the B.S. wave function has many independent components. This makes the numerical calculation difficult. However, when Coulomb gauge as well as the center-of-mass frame is adopted, the wave function becomes simple.

From equation (18), we find

$$
q_{i} \varphi_{i j}(P, \mathbf{q})=0
$$

This equation gives a very strong restriction on the wave functions. The following are some three dimensional wave functions.

For $0^{-+}$state(pseudoscalar)

$$
\varphi_{i j}(P, \mathbf{q})=f_{p}(\mathbf{q}) q_{k} \epsilon_{i j k}
$$

For $0^{++}$state(scalar)

$$
\varphi_{i j}(P, \mathbf{q})=f_{s}(\mathbf{q})\left(\delta_{i j}-\frac{q_{i} q_{j}}{\mathbf{q}^{2}}\right)
$$

For $2^{++}$state

$$
\varphi_{i j}(P, \mathbf{q})=f_{t 1}(\mathbf{q})\left(\delta_{i k}-\frac{q_{i} q_{k}}{\mathbf{q}^{2}}\right) \eta_{k l}\left(\delta_{j l}-\frac{q_{j} q_{l}}{\mathbf{q}^{2}}\right)+f_{t 2}(\mathbf{q})\left(\delta_{i j}-\frac{q_{i} q_{j}}{\mathbf{q}^{2}}\right) \eta_{k l} q_{k} q_{l} .
$$

For $2^{-+}$state

$$
\begin{aligned}
\varphi_{i j}(P, \mathbf{q})= & f_{p 1}(\mathbf{q}) \epsilon_{k l m} \eta_{m n} q_{n}\left(\delta_{i k}-\frac{q_{i} q_{k}}{\mathbf{q}^{2}}\right)\left(\delta_{j l}-\frac{q_{j} q_{l}}{\mathbf{q}^{2}}\right) \\
& +f_{p 2}(\mathbf{q})\left(\epsilon_{k m n} \eta_{m l} q_{n}-\epsilon_{l m n} \eta_{m k} q_{n}\right)\left(\delta_{i k}-\frac{q_{i} q_{k}}{\mathbf{q}^{2}}\right)\left(\delta_{j l}-\frac{q_{j} q_{l}}{\mathbf{q}^{2}}\right) \\
& +f_{p 3}(\mathbf{q}) q_{k} \epsilon_{i j k} \eta_{m n} q_{m} q_{n},
\end{aligned}
$$

where $\eta_{i j}$ is the polarization tensor of the glueball and $f_{p}, f_{s}, f_{t i}$ and $f_{p i}$ are scalar functions of $|\mathbf{q}|$.

Substituting equation (30) and the expression of kernel (21) and (25) into equation (18), we obtain an equation about $0^{-+}$state(For simplicity, in the following formulae, $|\mathbf{q}|$ and $|\mathbf{k}|$ are expressed simply by $q$ and $k$ respectively.)

$$
\begin{aligned}
& E\left(M^{2}-4 E^{2}\right) f_{p}(q)= \\
& -8 \pi \int \frac{d^{3} k}{(2 \pi)^{3}}\left\{\left[A(q, k) \frac{k}{q} \cos \theta+8 k^{2} \sin ^{2} \theta\right] V_{s}(q, k)+B(q, k) \frac{k}{q} \cos \theta V_{l}(q, k)\right\} f_{p}(k),
\end{aligned}
$$


where $\theta$ is the angle between vectors $\mathbf{q}$ and $\mathbf{k}$, and

$$
\begin{gathered}
A(q, k)=M^{2}+q^{2}+k^{2}+2 q k \cos \theta-\frac{\left(q^{2}-k^{2}\right)^{2}}{q^{2}+k^{2}-2 q k \cos \theta}, \\
B(q, k)=M^{2}+q^{2}+k^{2}+2 q k \cos \theta \\
V_{s}(q, k)=\frac{4 \pi}{3\left(q^{2}+k^{2}-2 q k \cos \theta\right) \ln \left(a+\frac{q^{2}+k^{2}-2 q k \cos \theta}{\Lambda_{Q C D}^{2}}\right)}, \\
V_{l}(q, k)=\frac{2 \lambda}{\left(q^{2}+k^{2}-2 q k \cos \theta\right)^{2}} .
\end{gathered}
$$

The equations for other states are given in the Appendix. We can see that for $0^{-+}$ state and $0^{++}$state the equations are simple because each wave function only has one component. However, for $2^{++}$state we have to solve two coupled integral equations. The appearance of the wave function of $2^{-+}$state is a little awful but the actual calculation shows that the three coupled equations about it are decoupled into two, so it is not too difficult to solve the equations.

3. Results and discussion There are three parameters in the present model: $a$ and $\Lambda_{Q C D}$ appearing in the running coupling constant and $\lambda$, the string tension. $\Lambda_{Q C D}$ is chosen as $200 \mathrm{MeV}$ which is commonly used in various models. The parameter $a$ is chosen as 4.0. Such a choice implies that the running coupling constant tends to its largest value 1.0 when the exchanged momentum goes to zero. We can relate the parameter $a$ to the threshold of the two massive gluon formation, and such a choice corresponds to a threshold of $400 \mathrm{MeV}\left(2 \Lambda_{Q C D}\right)$. As for the string tension, $\lambda$, it's different from the case of $q \bar{q}$ bound states where $\lambda$ is about $0.18(\mathrm{GeV})^{2}$. As argued in ref. [7], in the most naive picture, the string tension between two gluons should be twice that between two quarks because each gluon acts like a $q \bar{q}$ pair. Therefore, we chose $\lambda=0.36(\mathrm{GeV})^{2}$. Such a value has also been used in glueball investigation[11] and condensation calculation in QCD sum rules[12].

As most of the constituent models, in our investigation we give an effective mass to the constituent gluons despite the fact that gluons are massless in QCD Lagrangian. We can think that dynamical mass of gluons is generated through confinement interaction. 
One measure of the effective gluon mass is the energy $(\approx 2 m)$ necessary to break the string joining two color-octet sources to materialize a gluon pair. Such a question has been studied in a lattice calculation 13] with results $m \gtrsim 520 \mathrm{MeV}$. Non-perturbative continuum studies 14 also yield $m=(500 \pm 200) \mathrm{MeV}$. In our investigation we give the results when $m=(0,400,600) \mathrm{MeV}$.

With all the parameters determined, we solve the B.S. equation numerically. In actual calculation, the momentum integration must be cut at some value $\Lambda$, and when $\Lambda$ large enough, the results are independent of it. The results are given in table 1. For comparison, the recent lattice results are also given in the table.

We can see that our results are in good agreement with that of lattice calculation though there are some uncertainties due to the effective gluon mass. If we set the gluon mass to zero, the results favor that of the UKQCD group, while the results with a large gluon mass favor that of the GF11 group.

In the early investigation, a light $0^{-+}$glueball was preferred. For example, in MITBag model[6] $M\left(0^{-+}\right)=0.4 \mathrm{GeV}$, in potential model[7] $M\left(0^{-+}\right)=1.4 \mathrm{GeV}$, and in QCD sum rules [4], $M\left(0^{-+}\right)=1.7 \mathrm{GeV}$. However, recent lattice calculation indicates that $0^{-+}$is a very heavy particle[8], $M\left(0^{-+}\right)=2.3 \mathrm{GeV}$. Our investigation gives the same result as that on lattice.

In the nonrelativistic potential model. the form of the interaction between the two constituent gluons is similar to that of present paper, but their results are much saller. There are two reasons for this fact.Firt, the B.S. equation is a relativistic equation. More relativistic effects still survive in our model, even many approximations have been made. Second, the string tension is an important parameter. In our model, the choice $\lambda=0.36 \mathrm{GeV}^{2}$ is reasonable.

In fact, the effective mass of the gluon is still unclear. Someone has argued that the effective gluon mass is the result of confinement mechanism while the others think it incompatible with the principle of gauge invariance. It's interesting that our numerical results show that the glueball mass is quite insensitive to the effective mass of the gluons. We can even chose the gluon mass as zero without much change of the mass 
of the glueball.

It's known that nonrelativistic potential models are very successful in meson spectrum calculation for heavy quark systems. One of the reasons of the success is that most mesons studied can be considered as nonrelativistic systems because the mass of mesons is quite near the sum of the mass of its constituents. However things are different for glueballs. Notice the fact, that the glueball mass is much larger than the sum of the effective gluon mass. This means that glueballs are relativistic systems. To deal with such systems, perhaps, the B.S. equation is a more suitable method.

4.Acknowledgments. We wish to thank Prof. Yuan-Ben Dai and Prof. Xin-Heng Guo for interesting discussions.

This work was supported by Chinese National Science Foundation. 
Appendix

The B.S. equation for $0^{++}$state

$$
\begin{aligned}
E\left(M^{2}-4 E^{2}\right) f_{s}(q)= & -8 \pi \int \frac{d^{3} k}{(2 \pi)^{3}}\left\{\left[\frac{1}{2} A(q, k)\left(1+\cos ^{2} \theta\right)+4\left(q^{2}+k^{2}\right) \sin ^{2} \theta\right] V_{s}(q, k)\right. \\
& \left.+\frac{1}{2} B(q, k)\left(1+\cos ^{2} \theta\right) V_{l}(q, k)-\frac{3 \alpha_{s}}{2}\left(3-\cos ^{2} \theta\right)\right\} f_{s}(k) .
\end{aligned}
$$

The two coupled B.S. equation for $2^{++}$state

$$
\begin{aligned}
& E\left(M^{2}-4 E^{2}\right) f_{t 1}(q)=-8 \pi \int \frac{d^{3} k}{(2 \pi)^{3}}\left\{\left[\frac{1}{4} A(q, k)\left(1+\cos ^{2} \theta\right)^{2}-k^{2} \sin ^{4} \theta\right] V_{s}(q, k)\right. \\
& \left.+\frac{1}{4} B(q, k)\left(1+\cos ^{2} \theta\right)^{2} V_{l}(q, k)+\frac{3 \alpha_{s}}{4}\left(1+\cos ^{2} \theta\right)^{2}\right\} f_{t 1}(k) \\
& +8 \pi \int \frac{d^{3} k}{(2 \pi)^{3}}\left\{\left[\frac{1}{4} A(q, k) k^{2} \sin ^{4} \theta-2 k^{4} \sin ^{4} \theta\right] V_{s}(q, k)\right. \\
& \left.+\frac{1}{4} B(q, k) \sin ^{4} \theta V_{l}(q, k)+\frac{3 \alpha_{s}}{4} k^{2} \sin ^{4} \theta\right\} f_{t 2}(k), \\
& E\left(M^{2}-4 E^{2}\right) \quad f_{t 2}(q)=8 \pi \int \frac{d^{3} k}{(2 \pi)^{3}}\left\{\left[\frac{1}{8 q^{2}} A(q, k)\left(5 \operatorname{coc}^{4} \theta-6 \cos ^{2} \theta+1\right)\right.\right. \\
& \left.-\frac{k^{2}}{2 q^{2}}\left(5 \operatorname{coc}^{4} \theta-6 \cos ^{2} \theta+1\right)-2\left(3 \cos ^{4} \theta-5 \cos ^{2} \theta+2\right)\right] V_{s}(q, k) \\
& \left.+\frac{1}{8 q^{2}} B(q, k)\left(5 \operatorname{coc}^{4} \theta-6 \cos ^{2} \theta+1\right) V_{l}(q, k)+\frac{3 \alpha_{s}}{8 q^{2}}\left(5 \cos ^{4} \theta-18 \cos ^{2} \theta+5\right)\right\} f_{t 1}(k) \\
& -8 \pi \int \frac{d^{3} k}{(2 \pi)^{3}}\left\{\left[\frac{k^{2}}{8 q^{2}} A(q, k)\left(5 \cos ^{4} \theta+6 \cos ^{2} \theta-3\right)+2 k^{2}\left(3 \cos ^{2} \theta-1\right) \sin ^{2} \theta\right.\right. \\
& \left.+\frac{k^{4}}{q^{2}}\left(5 \cos ^{2} \theta-1\right) \sin ^{2} \theta\right] V_{s}(q, k)+\frac{k^{2}}{8 q^{2}} B(q, k)\left(5 \cos ^{4} \theta+6 \cos ^{2} \theta-3\right) V_{l}(q, k) \\
& \left.+\frac{3 \alpha_{s} k^{2}}{8 q^{2}}\left(5 \cos ^{4} \theta-18 \cos ^{2} \theta+5\right)\right\} f_{t 2}(k)
\end{aligned}
$$


The three coupled B.S. equation for $2^{-+}$state

$$
\begin{gathered}
E\left(M^{2}-4 E^{2}\right) \quad\left(f_{p 1}(q)+f_{p 2}(q)\right)=-8 \pi \int \frac{d^{3} k}{(2 \pi)^{3}}\left\{\left[\frac{k}{q} A(q, k) \cos \theta+8 k^{2} \sin ^{2} \theta\right] V_{s}(q, k)\right. \\
\left.+\frac{k}{q} \cos \theta B(q, k) V_{l}(q, k)\right\}\left(\cos ^{2} \theta-\frac{1}{2} \sin ^{2} \theta\right)\left(f_{p 1}(k)+f_{p 2}(k)\right) \\
-8 \pi \int \frac{d^{3} k}{(2 \pi)^{3}}\left\{\left[\frac{k^{3}}{q} A(q, k) \cos \theta \sin ^{2} \theta+2 k^{4} \sin ^{4} \theta\right] V_{s}(q, k)\right. \\
\left.+\frac{k^{3}}{q} A(q, k) \cos \theta \sin ^{2} \theta V_{l}(q, k)\right\} f_{p 3}(k), \\
E\left(M^{2}-4 E^{2}\right) \quad\left(f_{p 1}(q)-2 f_{p 2}(q)\right)=-8 \pi \int \frac{d^{3} k}{(2 \pi)^{3}}\left\{\left[\frac{k}{q} A(q, k) \cos \theta\right.\right. \\
\left.\left.+8 k^{2} \sin ^{2} \theta\right] V_{s}(q, k)+\frac{k}{q} \cos \theta B(q, k) V_{l}(q, k)\right\}\left(f_{p 1}(k)+f_{p 2}(k)\right) \\
-8 \pi \int \frac{d^{3} k}{(2 \pi)^{3}}\left\{\left[\frac{k^{3}}{q} A(q, k) \cos \theta \sin ^{2} \theta-4 k^{4} \sin ^{4} \theta\right] V_{s}(q, k)\right. \\
\left.+\frac{k^{3}}{q} A(q, k) \cos ^{2} \theta \sin ^{2} \theta V_{l}(q, k)\right\} f_{p 3}(k), \\
\quad f_{p 3}(q)=-8 \pi \int \frac{d^{3} k}{(2 \pi)^{3}}\left\{\left[\frac{k^{3}}{2 q^{3}} A(q, k)\left(5 \cos ^{3} \theta-3 \cos \theta\right)\right.\right. \\
\left.\quad-\frac{2 k^{4}}{q^{2}}\left(5 \cos ^{4} \theta-6 \cos ^{2} \theta+1\right)\right] V_{s}(q, k) \\
\left.+\frac{k^{3}}{2 q^{3}}\left(5 \cos ^{3} \theta-3 \cos ^{2} \theta\right) B(q, k) V_{l}(q, k)\right\} f_{p 3}(k) .
\end{gathered}
$$

where $\theta$ is the angle between vector $\mathbf{q}$ and $\mathbf{k}$, and the functions $A(q, k), B(q, k), V_{s}(q, k)$ and $V_{l}(q, k)$ have been defined in equation(35-38). 


\section{References}

[1] H. Fritzsch and Gell-Mann,Proc. 16 Intern. Conf. on High Energy Physics(FNAL, Batavia, IL, 1972);

[2] H.Fritzsch and Mikowski, Nuovo Cim. 30A(1975)393;

[3] V.A. Novikov, M.A. Shifman, A.I. Vainshtein and V.I. Zakharov, Nucl. Phys. B191(1981)301;

[4] S. Narison, Z. Phys. C26(1984)209;

[5] R.L. Jaffe and K. Johnson, Phys. Lett. B60 (1976)201;

[6] C. Detar and J.F. Donoghue, Ann. Rev. Nucl. Part. Sci. 33(1983)235;

[7] J.M. Cornwall and A. Soni, Phys. Lett. B120(1983)431;

[8] UKQCD Colloboration, G. Bali, K. Schilling, A. Hulsebos, A.C. Irving,C. Michael and P. Stephenson, Phys. Lett. B309(1993)378;

[9] H. Chen,J. Sexton, A. Vaccarino and D. Weingarten, Nucl. Phys. B(Proc. Suppl.) $34(1994) 357$;

[10] Y.B. Dai, C.S. Huang and H.Y. Jin, Phys. Lett. B331(1994)174;

[11] E.E. Boos, Phys. Lett. B193 (1987)301;

[12] B.A. Arbuzov, E.E. Boos and K.Sh. Turashvili, Z. Phys. C30(1986)287;

[13] C.Bernard, Phys. Lett. B108 (1982)431;

[14] J.M. Cornwall, UCLA preprint 81/TEP/12; UCLA preprint 81/TEP/30. 


\section{Figuer Caption}

Fig. 1: The diagrams contributing to the short distance part of the B.S. kernel. 


\section{Table Caption}

Table 1: The mass $(\mathrm{GeV})$ of the glueball states. 
Table 1. The mass of the glueballs $(\mathrm{GeV})$.

\begin{tabular}{|l|c|c|c|c|c|}
\hline & $M\left(0^{++}\right)$ & $M\left(0^{-+}\right)$ & $M\left(2^{++}\right)$ & $M\left(2^{-+}\right)$ & $R=\frac{M\left(2^{++}\right)}{M\left(0^{++}\right)}$ \\
\hline our results $(\mathrm{m}=0)$ & 1.50 & 2.18 & 2.17 & 2.18 & 1.45 \\
\hline our results $(\mathrm{m}=0.4)$ & 1.60 & 2.28 & 2.23 & 2.28 & 1.39 \\
\hline our results $(\mathrm{m}=0.6)$ & 1.75 & 2.39 & 2.32 & 2.39 & 1.33 \\
\hline Lattice results[8] & $1.550 \pm 0.050$ & - & $2.27 \pm 0.100$ & - & 1.46 \\
\hline Lattice results[9] & $1.740 \pm 0.071$ & - & $2.359 \pm 0.128$ & - & 1.36 \\
\hline
\end{tabular}

